\title{
Influence of the absorber dimensions on wavefront shaping based on volumetric optoacoustic feedback
}

\author{
X. Luís Deán-Ben, ${ }^{1, \text { * }}$ Héctor Estrada, ${ }^{1, \text { * }}$ Ali Ozbek, ${ }^{1}$ and Daniel Razansky ${ }^{1,2, \text { 丹 }}$ \\ ${ }^{1}$ Institute for Biological and Medical Imaging (IBMI), \\ Helmholtz Zentrum München, Neuherberg, Germany \\ ${ }^{2}$ School of Medicine, Technische Universität Mnchen (TUM), Munich, Germany
}

(Dated: September 29, 2018)

\begin{abstract}
The recently demonstrated control over light distribution through turbid media based on realtime three-dimensional optoacoustic feedback has offered promising prospects to interferometrically focus light within scattering objects. Nevertheless, the focusing capacity of the feedback-based approach is strongly conditioned by the number of effectively resolvable optical modes (speckles). In this letter, we experimentally tested the light intensity enhancement achieved with optoacoustic feedback measurements from different sizes of absorbing microparticles. The importance of the obtained results is discussed in the context of potential signal enhancement at deep locations within a scattering medium where the effective speckle sizes approach the minimum values dictated by optical diffraction.
\end{abstract}

Light scattering in nanoscale heterogeneities is responsible for the optical opacity of materials, in particular biological tisues [1]. Even though photons can generally penetrate scattering tissues up to a depth of several centimeters [2], progressive changes in their propagation direction impede the ability to trace the exact trajectory of light and hence see through this type of samples. The light intensity distribution caused by photon interference is however not entirely random but determined by the type and location of optical scatterers, which results in a characteristic speckle pattern [3]. By controlling the incident light wavefront, it becomes possible that photons positively interfere at specific locations, thus making focusing through scattering objects theoretically feasible. Wavefront shaping techniques have recently emerged as a promising tool to image behind strongly scattering samples, with a handful of potential applications foreseen [49]. Of particular importance is the possibility to image deep into biological tissues, where scattering limits the penetration of the modern optical microscopy approaches to a depth of a few hundred microns [10]. In the socalled near-infrared window, diffuse light can penetrate up to several centimeters into highly scattering tissues [2], thus wavefront-shaping-based techniques can potentially enable high-resolution bio-optical imaging at centimeterscale depths, ultimately limited by light absorption only.

Wavefront shaping methods are based on the spatial modulation of the phase of the incoming light, which is commonly done by means of a spatial light modulator (SLM) device [1]. The optimal phase mask at the SLM can be determined from readings of the light intensity at the target point(s). The maximum signal enhancement $\eta$ at a given point (focus) depends on the spatial resolution of the feedback method via the following relation [1]

$$
\eta=\frac{N_{\mathrm{SLM}}}{2 N_{\text {modes }}},
$$

where $N_{\text {modes }}$ is the number of optical modes generating the measured signal and $N_{\text {SLM }}$ is the number of degrees of freedom (pixels) of the SLM. The number of optical modes generally refers to the number of speckles enclosed in the area (or volume) that can be effectively resolved with the technique employed for measuring the light intensity.

The feasibility to focus through scattering objects with wavefront shaping techniques was first demonstrated by using the two-dimensional image acquired with a CCD camera as a means to determine the light energy distribution [4]. Application of this approach to fluorescence microscopy was further showcased [5]. Optical approaches are however not suitable for providing an efficient feedback on the light distribution inside scattering objects as the spatial resolution quickly deteriorates in the diffuse propagation regime [10]. Thereby, alternative approaches based on a combination of optics and ultrasound have been recently suggested as a means to provide high resolution feedback from a scattering medium. For instance, the frequency (wavelength) of the light beam can be modulated at a given location with focused ultrasound so that the phase of the modulated wavefront can be holographically recorded after propagation through the object. In this case, by means of the phase conjugation, the light beam can be directed to a certain ultrasonically-tagged position 12 14. Alternatively, wavefront shaping based on the optoacoustic feedback may offer important advantages to control the scattered light distribution. In this approach, the feedback signals can be in principle collected at a very high rate, ultimately only limited by the ultrasound propagation speed, and hence provide a very fast control mechanism over SLM mask optimization. Acquisition of signals with transducer arrays combined with graphics processing unit (GPU)-based reconstruction procedures further enable real time image rendering in two- and three dimensions [15], which can even exceed the currently available refresh rates of standard SLMs [16]. Thereby, both single-element transducers and 
transducer arrays have been suggested for optoacoustic wavefront shaping $[17$ 21]. Moreover, the high optoacoustic resolution enables a high light intensity enhancement according to Eq. 1, which can be further enhanced by conveniently selecting the detection frequency band [22] or by using non linear effects [23]. Finally, light absorption essentially occurs in any substance in nature, which makes optoacoustics arguably the most versatile feedback approach in terms if imaging contrast.

Typically, the photothermal energy conversion leading to optoacoustic signal generation takes place in light absorbing molecules. The size and average separation between the molecules is generally much smaller than the speckle dimensions. For example, organic molecules have typical lengths of a few nanometers, while the minimal speckle size is determined by the optical diffraction limit. i.e. $\lambda / 2$, where $\lambda$ is the wavelength. For more than one absorber per speckle, $N_{\text {modes }}$ in Eq. 1 refers to the number of speckles in the region that can be resolved with the method employed to measure the light intensity. However, larger particles sparsely distributed across the region of interest can also be used to generate the optoacoustic signals, in which case the number of speckles may be generally higher than the number of absorbers. In this case, $N_{\text {modes }}$ in Eq. 1 is given by

$$
N_{\text {modes }}=\sum_{i=1}^{N} N_{s p, i},
$$

being $N$ the number of particles enclosed in the resolved region and $N_{s p, i}$ the number of optical speckles in each particle. The ratio between the sizes of the speckles and the particles becomes then a crucial factor in determining the potential enhancement achieved, which is also conditioned by the number of particles required to achieve detectable optoacoustic signal levels. Proper selection of the absorber characteristics is therefore of key importance for achieving favorable wavefront shaping results with the optoacoustic feedback approach.

A previously reported volumetric (three-dimensional) optoacoustic wavefront shaping platform 21] was used herein to experimentally investigate the effects of the particle size on the light intensity enhancement. The lay-out of the set-up employed is depicted in Fig. 1. Basically, a phase-only SLM consisting of a liquid crystal on Silicon (LCoS) microdisplay with $8 \mu \mathrm{m}$ pixel pitch (PLUTO-BB II, Holoeye Photonics AG) was used to control the light wavefront distribution after passing through a ground glass diffuser (Thorlabs DG10-120). The light enhancement was targeted at polyethylene microparticles with approximate diameters $100 \mu \mathrm{m}$ (Cospheric BKPMS 90-106) and $200 \mu \mathrm{m}$ (Cospheric BKPMS 180-210) as well as Carbon microspheres with a diameter around $400 \mu \mathrm{m}$ (SPI-Supplies) embedded into an agar phantom. The microspheres were excited with the output beam of a frequency-doubled Q-switch Nd:YAG laser

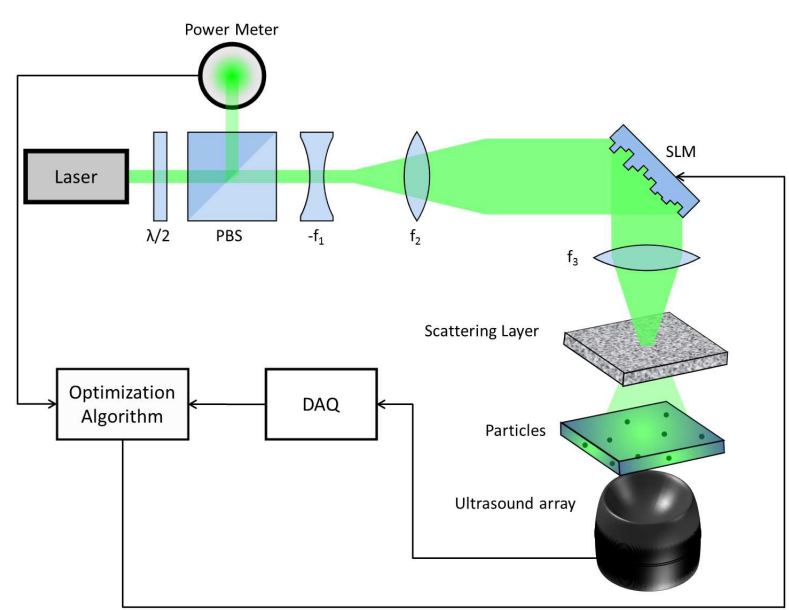

FIG. 1. Lay-out of the experimental set-up.
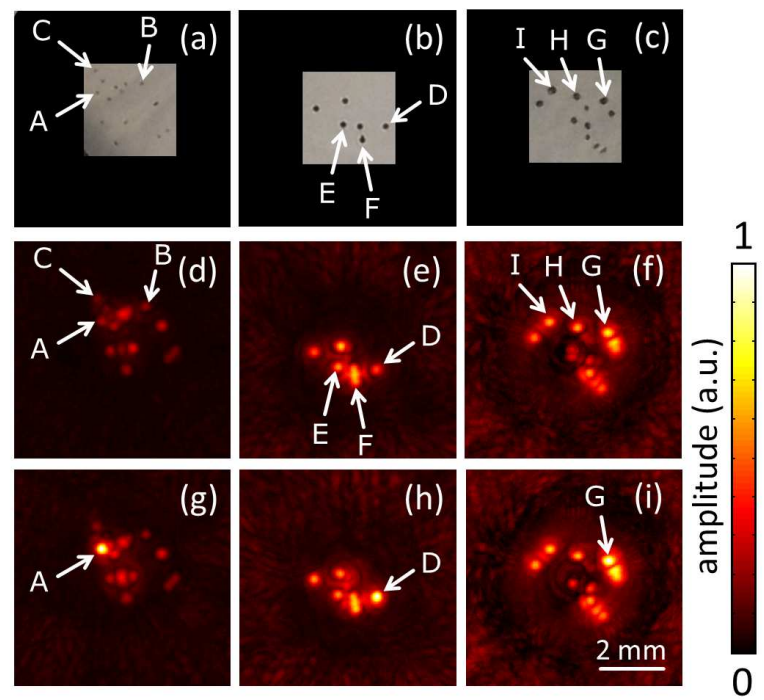

FIG. 2. (a-c) Photographs of the agar-embedded microparticles with diameters $100 \mu \mathrm{m}, 200 \mu \mathrm{m}$ and $400 \mu \mathrm{m}$, respectively; (d-f) Optoacoustic images of the corresponding phantoms obtained with a constant phase value of the SLM pixels (initial iteration). Maximum intensity projections along the depth direction are shown; (g-i) Corresponding optoacoustic images obtained after the phase of the SLM pixels was optimized to deliver maximum light fluence to the particles labelled 'A', 'D' and 'G' respectively.

(Lab-190-30, Spectral Physics) operating at 15 pulses per second, which was collimated and horizontally polarized before being directed onto the SLM. The generated optoacoustic signals were simultaneously collected with a spherical array of piezoelectric transducers [24], while the three-dimensional optoacoustic images representing the light absorption distribution in the phantom were reconstructed for each laser pulse using a graphics processing unit (GPU) [15]. 
The reconstructed image was used as a feedback mechanism for a genetic algorithm that optimizes the phase mask at the pixels of the SLM based on the maximization of a defined cost function [21]. In the experiments, a mask of 20x20 phase values was optimized by grouping the SLM pixels accordingly. The speckle diameter was set to approximately $27 \mu \mathrm{m}$ by controlling the distance between the particles and the diffuser. The cost function was defined as the maximum image value in the volume of interest (VOI) enclosing a certain microsphere, such that the genetic algorithm converges to an SLM mask that focuses light at this particular absorber. The genetic algorithm performs the optimization as described in [18]. Specifically, a population of 20 phase masks evolves with a refresh rate of $25 \%$ and a mutation rate that ranges between $10 \%$ and $1.25 \%$ of the pixels of the phase mask.

Actual photographs of the phantoms are depicted in Figs. 2a-c for microspheres with diameters 100, 200 and $400 \mu \mathrm{m}$, respectively. The maximum intensity projections (MIP) along the depth direction of the corresponding reconstructed optoacoustic images are subsequently shown in Figs. 2d-f. The MIPs in Figs. 2d-f are obtained by setting a constant phase value in the SLM, which represents the initial iteration of the genetic algorithm. The corresponding MIPs of the images rendered after 2500 iterations of the genetic algorithm are displayed in Figs. $2 \mathrm{~g}-\mathrm{i}$ for cost functions defined to maximize the light intensity on particles labeled A, D and G, respectively. The evolution of the cost function (maximum signal intensity) as a function of the number of iterations in the genetic algorithm for all particles labeled in Fig. 2 is displayed in Figs. 3a-c. Fig. 3d shows the signal enhancement for all the imaged particles as a function of their diameter. The enhancement is estimated as the maximum value of the cost function for the given particle after normalizing it to the average value for the first 20 iterations, which correspond to the initial random population of the genetic algorithm. The small enhancement for the $400 \mu \mathrm{m}$ particles can be attributed to signal fluctuations approaching the noise levels, so that it was not possible to focus light in those cases. As expected, the enhancement is maximized for smaller particles containing a lower number of speckles.

The results showcased indicate the importance of the absorber dimensions used to provide feedback in optoacoustics-based wavefront shaping, which ultimately determines the focusing (light enhancement) capacity of this approach. The maximum signal enhancement achieved is of particular relevance when eventually aiming at focusing light inside scattering samples, e.g. biological tissues, where the speckles at depths beyond the transport mean free path are expected to have characteristic lengths approaching $\lambda / 2$. In order to generate efficient feedback from absorbers in the sub-micron size range, the optoacoustic imaging system would need to have an efficient detection bandwidth in the hundreds of
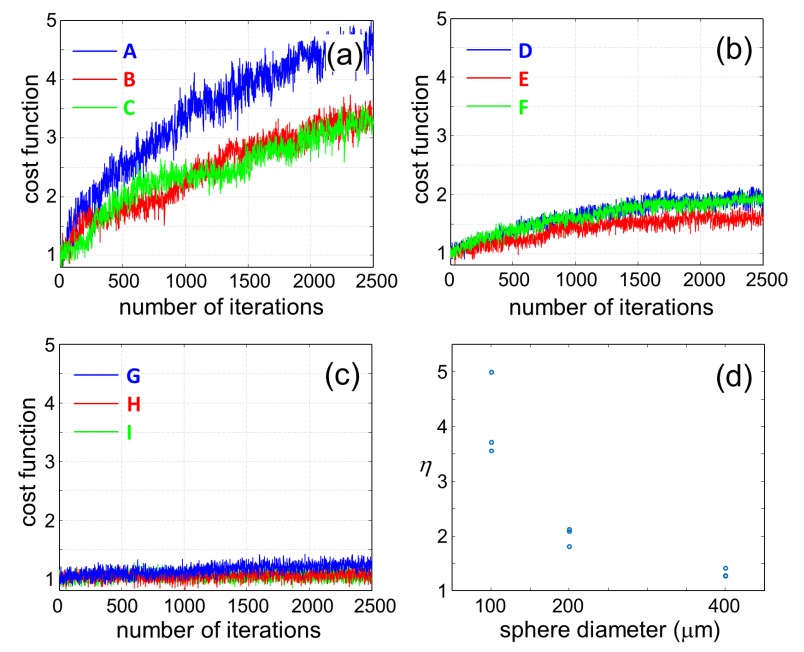

FIG. 3. Value of the cost function as a function of the number of iterations in the genetic algorithms for the particles with diameter $100 \mu \mathrm{m}$ (a), $200 \mu \mathrm{m}$ (b) and $400 \mu \mathrm{m}$ (c). The light intensity enhancements achieved as a function of the particle diameter are displayed in (d).

$\mathrm{MHz}$ range. However, the image artifacts and resolution degradation associated with acoustic heterogeneities and attenuation of the high frequency components in the imaged medium are expected to impose hard limitations on the achievable spatial resolution when using the optoacoustic imaging feedback. In biomedical optoacoustic imaging, the practically achievable resolution is generally in the range of $1 / 200$ of the imaging depth [25]. Thus, the realistically achievable spatial resolution at a depth of $5 \mathrm{~mm}$ is expected to be in the range of $25 \mu \mathrm{m}$. As a result, approximately 250000 speckles will be accommodated in each resolution-limited voxel, for which no significant light intensity enhancement is feasible. Higher efficiency of the light enhancement can be potentially accomplished when properly selecting the absorbing particles and their concentration so that a relatively low number of light speckles is enclosed within the resolved volume while simultaneously attaining sufficient detection sensitivity. Yet, focusing light within biological tissues still remains a challenging topic due to additional factors, such as the millisecond-level speckle decorrelation times.

In conclusion, the size of light absorbing particles is designated to play an essential role in volumetric wavefront shaping using optoacoustic feedback. The experimental analysis performed in this work may help to provide general guidelines for optimizing the particle size for achieving efficient light enhancement at locations exhibiting small speckle size, e.g. beyond the transport mean free path depth within turbid tissues.

This project was supported in part by the European Research Council through the grant agreement ERC- 
2010-StG-260991.

* X.L.D.B and H.S contributed equally to this work † Corresponding author: dr@tum.de

[1] Allard P Mosk, Ad Lagendijk, Geoffroy Lerosey, and Mathias Fink. Controlling waves in space and time for imaging and focusing in complex media. Nature photonics, 6(5):283-292, 2012.

[2] Lihong V Wang and Hsin-i Wu. Biomedical optics: principles and imaging. John Wiley \& Sons, 2012.

[3] Joseph W Goodman. Speckle phenomena in optics: theory and applications, volume 1. 2007.

[4] Ivo M Vellekoop and AP Mosk. Focusing coherent light through opaque strongly scattering media. Optics letters, 32(16):2309-2311, 2007.

[5] Ivo M Vellekoop and Christof M Aegerter. Scattered light fluorescence microscopy: imaging through turbid layers. Optics letters, 35(8):1245-1247, 2010.

[6] David J McCabe, Ayhan Tajalli, Dane R Austin, Pierre Bondareff, Ian A Walmsley, Sylvain Gigan, and Béatrice Chatel. Spatio-temporal focusing of an ultrafast pulse through a multiply scattering medium. Nature communications, 2:447, 2011.

[7] Ori Katz, Eran Small, Yaron Bromberg, and Yaron Silberberg. Focusing and compression of ultrashort pulses through scattering media. Nature photonics, 5(6):372$377,2011$.

[8] Ori Katz, Eran Small, Yefeng Guan, and Yaron Silberberg. Noninvasive nonlinear focusing and imaging through strongly scattering turbid layers. Optica, 1(3):170-174, 2014.

[9] Marco Leonetti, Salman Karbasi, Arash Mafi, and Claudio Conti. Light focusing in the anderson regime. Nature communications, 5, 2014.

[10] Vasilis Ntziachristos. Going deeper than microscopy: the optical imaging frontier in biology. Nature methods, 7(8):603-614, 2010.

[11] SM Popoff, Geoffroy Lerosey, Mathias Fink, Albert Claude Boccara, and Sylvain Gigan. Controlling light through optical disordered media: transmission matrix approach. New Journal of Physics, 13(12):123021, 2011.

[12] Xiao Xu, Honglin Liu, and Lihong V Wang. Timereversed ultrasonically encoded optical focusing into scattering media. Nature photonics, 5(3):154-157, 2011.

[13] Ke Si, Reto Fiolka, and Meng Cui. Fluorescence imaging beyond the ballistic regime by ultrasound-pulse-guided digital phase conjugation. Nature photonics, 6(10):657$661,2012$.

[14] Benjamin Judkewitz, Ying Min Wang, Roarke Horstmeyer, Alexandre Mathy, and Changhuei Yang.
Speckle-scale focusing in the diffusive regime with time reversal of variance-encoded light (trove). Nature photonics, 7(4):300-305, 2013.

[15] X Luís Dean-Ben, Ali Ozbek, and Daniel Razansky. Volumetric real-time tracking of peripheral human vasculature with gpu-accelerated three-dimensional optoacoustic tomography. Medical Imaging, IEEE Transactions on, 32(11):2050-2055, 2013.

[16] X L Deán-Ben, S J Ford, and D Razansky. Highframe rate four dimensional optoacoustic tomography enables visualization of cardiovascular dynamics and mouse heart perfusion. Scientific Reports, 2015. DOI:10.1038/srep10133.

[17] Fanting Kong, Ronald H Silverman, Liping Liu, Parag V Chitnis, Kotik K Lee, and Ying-Chih Chen. Photoacoustic-guided convergence of light through optically diffusive media. Optics letters, 36(11):2053-2055, 2011.

[18] Antonio M Caravaca-Aguirre, Donald B Conkey, Jacob D Dove, Hengyi Ju, Todd W Murray, and Rafael Piestun. High contrast three-dimensional photoacoustic imaging through scattering media by localized optical fluence enhancement. Optics express, 21(22):26671-26676, 2013.

[19] Thomas Chaigne, Ori Katz, A Claude Boccara, Mathias Fink, Emmanuel Bossy, and Sylvain Gigan. Controlling light in scattering media non-invasively using the photoacoustic transmission matrix. Nature Photonics, 8(1):58 64, 2014.

[20] Thomas Chaigne, Jérôme Gateau, Ori Katz, Emmanuel Bossy, and Sylvain Gigan. Light focusing and twodimensional imaging through scattering media using the photoacoustic transmission matrix with an ultrasound array. Optics letters, 39(9):2664-2667, 2014.

[21] X Luís Deán-Ben, Héctor Estrada, and Daniel Razansky. Shaping volumetric light distribution through turbid media using real-time three-dimensional opto-acoustic feedback. Optics letters, 40(4):443-446, 2015.

[22] Thomas Chaigne, Jérôme Gateau, Ori Katz, Claude Boccara, Sylvain Gigan, and Emmanuel Bossy. Improving photoacoustic-guided optical focusing in scattering media by spectrally filtered detection. Optics letters, 39(20):6054-6057, 2014.

[23] Puxiang Lai, Lidai Wang, Jian Wei Tay, and Lihong V Wang. Photoacoustically guided wavefront shaping for enhanced optical focusing in scattering media. Nature Photonics, 2015.

[24] Xosé Luís Deán-Ben, Erwin Bay, and Daniel Razansky. Functional optoacoustic imaging of moving objects using microsecond-delay acquisition of multispectral threedimensional tomographic data. Scientific reports, 4, 2014.

[25] Lihong V Wang and Song Hu. Photoacoustic tomography: in vivo imaging from organelles to organs. Science, 335(6075):1458-1462, 2012. 